\title{
Mending broken hearts
}

Adult mammalian hearts are virtually devoid of regenerative capacity. The mechanisms responsible for this extreme resistance to regeneration are poorly understood and strategies to boost cardiac regeneration have shown very limited benefits. Two recent studies offer considerable advancements in the understanding and management of heart repair.

In contrast to mammals, hearts of some lower vertebrates, including zebrafish, have high regenerative potential. Whereas mammalian cardiomyocytes are mainly polyploid, zebrafish cardiomyocytes are diploid. González-Rosa et al. investigated whether this difference in ploidy explains the discrepancy in regenerative capacity. To induce polyploidization in zebrafish cardiomyocytes, they

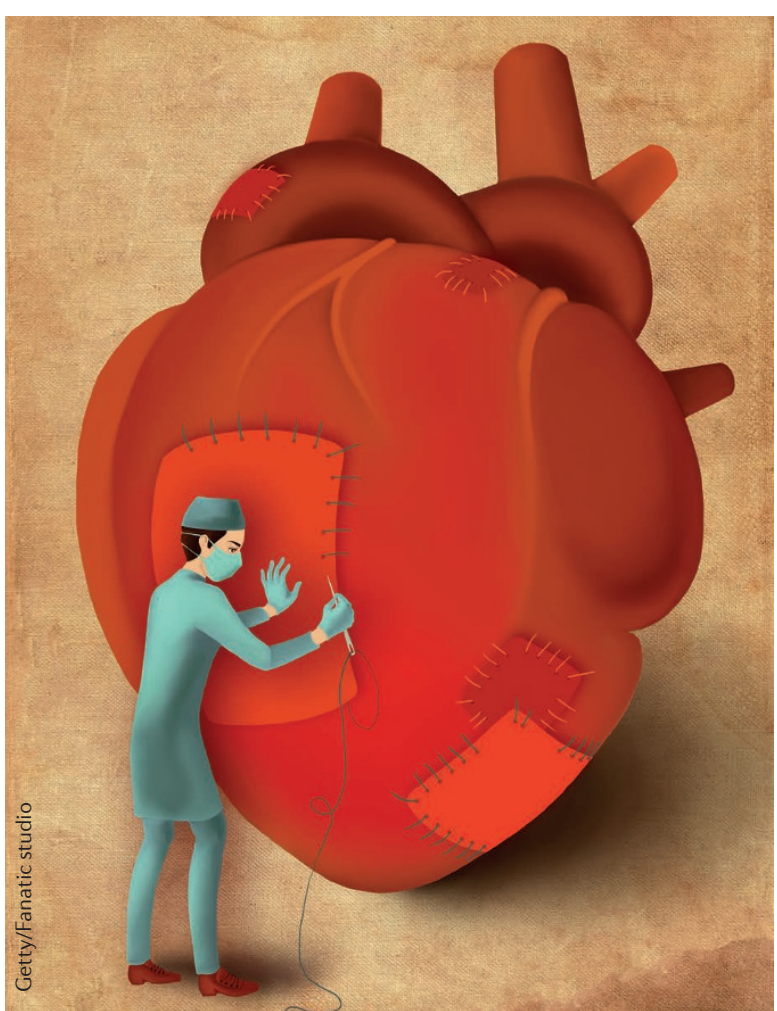

inhibited cytokinesis by introducing a dominant negative form of Ect2 (dnEct2), a protein that regulates cytokinetic ring assembly. Cardiacspecific expression of dnEct 2 caused cardiomyocyte polyploidization in zebrafish larvae, which was associated with impairment of heart function and of animal survival at later developmental stages.

In order to study regeneration of adult zebrafish hearts containing polyploid cardimyocytes, the authors generated zebrafish with heat shock-inducible expression of dnEct2. They induced heat shock daily between late larval stages and adulthood, yielding adult zebrafish with myocardium comprising $25 \%$ to $\sim 50 \%$ polyploid cardiomyocytes. Cellular and organismal fitness were unaltered in these animals. However, following cardiac injury, polyploid cell progeny were considerably underrepresented in regenerated tissue, and hearts with large amounts of polyploid cells ( $50 \%)$ did not regenerate, forming a scar instead. Thus, polyploidization of cardiomyocytes in zebrafish interferes with their otherwise robust proliferative capacity during heart regeneration.

In a screen for cell cycle regulators of cardiomyocytes, Mohamed et al. identified regulators of the G2/M transition - cyclin-dependent kinase 1 (CDK1), cyclin B (CCNB) and aurora kinase $\mathrm{B}-$ as factors that enhance proliferation of adult mouse cardiomyocytes. However, increased proliferation in response to the expression of these factors was associated with cell death, suggesting that these factors cause premature and/or aberrant entry into mitosis. The authors eventually established that co-expression of G1 phase regulators CDK4 and cyclin D1 (CCND) together with CDK1 and CCNB (a combination referred to as $4 \mathrm{~F}$ ) allows enhanced proliferation of cardiomyocytes while retaining cell viability.

Adenoviral delivery of 4F directly into the hearts of adult mice after heart infarction increased the number of dividing cardiomyocytes from $<1 \%$ to $\sim 16 \%$, as assessed by lineage tracing 4 days after infarction. Various parameters of heart function were improved at 12 weeks post-infarction in these mice. The improvement in heart function was observed even when $4 \mathrm{~F}$ was introduced 1 week after infarction, showing potential for the application of this strategy in a clinically relevant setting. Finally, the authors provided a modification of their protocol, whereby ectopic expression of G1-promoting factors CDK4 and CCND was accompanied by chemical inhibition of WEE1, an inhibitor of entry into mitosis, and of transforming growth factor $\beta$, which dampens the activity of CDK inhibitor p27. Overall, this study offers a strategy to efficiently modulate the cell cycle of adult cardiomyocytes to fuel their proliferation and heart repair after injury.

Moving forward, it would be interesting to understand whether the enhanced cardiomyocyte proliferation in response to cell cycle modulation is related to changes in their ploidy. Further steps would be to explore strategies to modulate the cell cycle and ploidy in human cardiomyocytes and eventually in human patients.

Paulina Strzyz

ORIGINAL ARTICLES González-Rosa, J. M. et al. Myocardial polyploidization creates a barrier to heart regeneration in zebrafish. Dev. Cell 44, 433-446.e7 (2018) | Mohamed, T. M. A. et al. Regulation of cell cycle to stimulate adult cardiomyocyte proliferation and cardiac regeneration. Cell https://doi.org/10.1016/ j.cell.2018.02.014 (2018) 